\title{
A critical role for Clock
}

the loss
of Clock
expression
delayed the
onset of
critical period
plasticity

5

Early postnatal life constitutes a 'critical period' during which the development and maturation of cortical neuronal circuits can be altered by sensory experience. This critical period of plasticity is thought to be driven by the appearance and maturation of parvalbumin (PV) interneuron networks; however, little is known about the underlying mechanisms. Kobayashi et al. now reveal a previously unknown role for circadian genes in the formation of functional interneuron networks and the determination of the critical period in the mouse visual cortex.

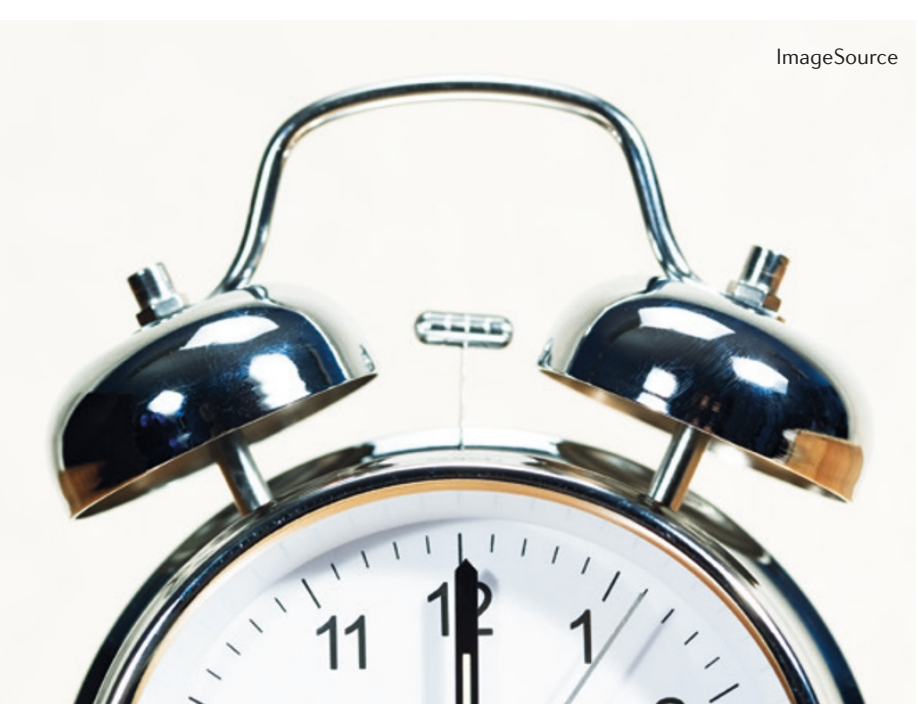

Circadian fluctuations in the expression of 'molecular clock' genes occur in all brain regions, including the cortex, but little is known about the roles of these circadian genes outside the suprachiasmatic nucleus. The authors found that oscillations in the expression of several key circadian genes emerged with the development of cortical circuitry and the onset of the critical period in the primary visual cortex (V1) in mice.

To determine whether there is a functional relationship between circadian genes and cortical circuit development, the authors compared cortical plasticity in mice lacking the key circadian gene Clock (Clock ${ }^{-1}$ mice) with that in control mice. Four days of monocular deprivation during the critical period (which begins around postnatal day 25 (P25) and lasts approximately 1 month) decreased the visual acuity of the deprived eye in control mice but not $\mathrm{Clock}^{-/-}$mice. By contrast, when monocular deprivation was carried out in adult mice (in which the critical period had ended in control mice) Clock $^{-1-}$ mice exhibited changes in visual acuity. Thus, the loss of Clock expression delayed the onset of critical period plasticity.

Critical period initiation coincides with the maturation of interneuron networks in V1 and its closure is thought to be governed by the formation of perineuronal nets (PNNs) of extracellular matrix proteins around their cell bodies. The authors found that Clock $^{-1-}$ mice exhibited reduced PV expression and fewer PV-positive neurons in V1, as well as a delay in the formation of PNNs. The specific ablation of Clock expression in PV-positive neurons recapitulated the delayed critical period observed in global $\mathrm{Clock}^{-/-}$mice, confirming a cell-autonomous role for the gene in these cells.

This study shows that the circadian gene Clock is important for the maturation of interneuron circuits in the visual cortex, and thus drives the onset and closure of critical period plasticity in this region. A microarray analysis revealed changes in genes associated with multiple aspects of synaptic and cellular function in $\mathrm{Clock}^{-/-}$cells. Further work will therefore be required to determine which of these pathways are important for the mis-timing of critical period plasticity in these mice.

Katherine Whalley

ORIGINAL RESEARCH PAPER Kobayashi, Y., Ye, Z. \& Hensch, T. K. Clock genes control cortical critical period timing. Neuron http://dx.doi. org/10.1016/j.neuron.2015.02.036 (2015) 